\title{
COMPLEX BORDISM OF CLASSIFYING SPACES
}

\author{
PETER S. LANDWEBER ${ }^{1}$
}

ABSTRACT. It is shown that, for a finite group $G$, the Thom homomorphism maps the complex bordism $\Omega_{*}^{U}\left(B_{G}\right)$ onto the homology $H_{*}\left(B_{G} ; Z\right)$ of a classifying space for $G$ if and only if $G$ has periodic cohomology.

Let $B_{G}$ denote a classifying space for the finite group $G$, and let

$$
\mu: \Omega_{*}^{U}\left(B_{G}\right) \rightarrow H_{*}\left(B_{G} ; Z\right)
$$

denote the Thom homomorphism ([5], [7]) from the complex bordism of $B_{G}$ to its integral homology. It is well known (e.g. see [9]) that $\mu$ is onto if $G$ is cyclic, and more generally if $G$ has periodic cohomology [4, Chapter XII, §11], since then $H^{n}\left(B_{G} ; Z\right)=0$ for all odd $n$, thus $H_{p}\left(B_{G}, \boldsymbol{Z}\right)=0$ for all even $p>0$ and this makes the complex bordism spectral sequence collapse. The main result of this note is the converse statement.

THEOREM 1. If $G$ is a finite group for which $\mu: \Omega_{*}^{U}\left(B_{G}\right) \rightarrow H_{*}\left(B_{G} ; Z\right)$ is onto, then $G$ has periodic cohomology.

The main step in the proof of this result has recently been supplied by R. G. Swan [11].

Theorem 2. A finite group $G$ with $H^{n}\left(B_{G} ; Z\right)=0$ for all odd $n$ must have periodic cohomology.

We state the next result in order to give a more complete picture. For a CW-complex $X$ write hom $\operatorname{dim}_{\Omega^{U}} \Omega_{*}^{U}(X)$ for the projective dimension of the $\Omega_{*}^{U}$-module $\Omega_{*}^{U}(X)$ [7].

THEOREM 3. The following conditions are equivalent for a finite group $G$ :

(a) $G$ has periodic cohomology, i.e. every abelian subgroup of $G$ is cyclic [4, Chapter XII, \$11].

(b) $H^{n}\left(B_{G} ; Z\right)=0$ for all odd $n$.

(c) The Atiyah spectral sequence [2] $H^{*}\left(B_{G} ; Z\right) \Rightarrow K^{*}\left(B_{G}\right)=(R(G))^{\wedge}$ collapses.

Received by the editors March 10, 1970.

AMS 1969 subject classifications. Primary 5530; Secondary 5536.

Key words and phrases. Complex bordism, classifying spaces, Thom homomorphism, periodic cohomology.

1 Research supported in part by NSF grant GP-9452 at Yale University. 
(d) The Thom homomorphism $\mu: \Omega_{*}^{U}\left(B_{G}\right) \rightarrow H_{*}\left(B_{G} ; Z\right)$ is onto.

(e) hom $\operatorname{dim}_{\mathbb{Q} U_{*}} \Omega_{*}^{U}\left(B_{G}\right) \leqq 1$.

The implication (a) $\rightarrow$ (b) follows by reduction to the Sylow subgroups and a computation [4, Chapter XII]. The implications (b) $\rightarrow$ (c) and (b) $\rightarrow$ (d) are well-known consequences of the vanishing of the even differentials in the $K$-theory and complex bordism spectral sequences. Furthermore the implication (c) $\rightarrow$ (b) follows from the facts [2] that $K^{1}\left(B_{G}\right)=0$ and that the $K$-theory filtration is even. The equivalence of (d) and (e) follows from Proposition 4 below.

We turn to the proof of Theorem 1. If the Thom homomorphism maps $\Omega_{*}^{U}\left(B_{G}\right)$ onto $H_{*}\left(B_{G} ; \boldsymbol{Z}\right)$, then the complex bordism spectral sequence

$$
H_{*}\left(B_{G} ; \Omega_{*}^{U}\right) \Rightarrow \Omega_{*}^{U}\left(B_{G}\right)
$$

collapses. Let $K_{*}()$ denote the $\boldsymbol{Z}_{2}$-graded homology theory dual to $K$-theory. J. W. Vick [12] has proved that $K_{1}\left(B_{G}\right)$ is isomorphic to the character group of the compact group $\widetilde{K}^{0}\left(B_{G}\right)$, and that $\widetilde{K}_{0}\left(B_{G}\right)$ $=0$. There is the $K_{*}$-spectral sequence

$$
H_{*}\left(B_{G} ; Z\right) \Rightarrow K_{*}\left(B_{G}\right),
$$

and we claim that it collapses if (1) does.

The natural transformation $\mu_{c}: \Omega_{*}^{U}() \rightarrow K_{*}()$ dual to the corresponding transformation from complex cobordism to $K$-theory [6] induces a morphism of the spectral sequence (1) into the spectral sequence (2). Since on the coefficient rings $\mu_{c}$ is the Todd genus and so maps $\Omega_{*}^{U}$ onto $K_{*}(p t)=\boldsymbol{Z}$, (2) indeed collapses if (1) does.

In the spectral sequence (2) we have $E_{p}^{2}=H_{p}\left(B_{G} ; Z\right)$ and

$$
E_{p}^{\infty} \cong \operatorname{Im}\left(K_{p}\left(B_{G}^{p}\right) \rightarrow K_{p}\left(B_{G}\right)\right) / \operatorname{Im}\left(K_{p}\left(B_{G}^{p-1}\right) \rightarrow K_{p}\left(B_{G}\right)\right),
$$

where $B_{G}^{k}$ is the $k$-skeleton of the complex $B_{G}$, and thus $E_{p}^{\infty}=0$ for even $p>0$. If (2) collapses then $E^{2}=E^{\infty}$ and so $H_{p}\left(B_{G} ; \boldsymbol{Z}\right)=0$ for all even $p>0$. By the universal coefficient theorem it follows that $H^{n}\left(B_{G} ; \boldsymbol{Z}\right)=0$ for all odd $n$, and then Theorem 1 is a direct consequence of Theorem 2 .

REMARK 1. A rapid but indirect proof that a finite $p$-group $G$ with $H^{n}\left(B_{G} ; Z\right)=0$ for all odd $n$ must have periodic cohomology can be based on Lemma (2.1) and Proposition (6.1) of [10]. Then $G$ is either a cyclic or generalized quaternion group.

REMARK 2. The same argument shows that the Thom homomorphism $\mu: \Omega_{U}^{*}\left(B_{G}\right) \rightarrow H^{*}\left(B_{G} ; Z\right)$ from complex cobordism to cohomology 
is onto if and only if $G$ has periodic cohomology. In fact, if $X$ is any complex and if either of the spectral sequences

$$
H^{*}(X ; Z) \Rightarrow K^{*}(X), \quad H^{*}\left(X ; \Omega_{U}^{*}\right) \Rightarrow \Omega_{U}^{*}(X)
$$

collapses, then so does the other. This observation is due to T. tom Dieck $[8, \S 6]$, and requires the theorem of Hattori and Stong; the homology analogue is also true.

The equivalence of (d) and (e) in Theorem 3 is a consequence of the next result.

Proposition 4. For any CW-complex $X, \mu: \Omega_{*}^{U}(X) \rightarrow H_{*}(X ; Z)$ is onto if and only if hom $\operatorname{dim}_{\Omega_{*}} \Omega_{*}^{U}(X) \leqq 1$.

For finite complexes the proof is given in $[7, \S 3]$. The following argument was suggested by Professor Larry Smith for the general case. We may regard $X$ as a CW-spectrum in J. M. Boardman's stable category [3], and then $X$ is $(-1)$-connected.

Lemma 5. Let $X$ be an n-connected CW-spectrum. Then there exists a cofibration $A_{1} \rightarrow A_{0} \rightarrow X$ of $n$-connected CW-spectra such that $\Omega_{*}^{U}\left(A_{0}\right)$ is a free $\Omega_{*}^{U}$-module and the sequence of induced homomorphisms

$$
0 \rightarrow \Omega_{*}^{U}\left(A_{1}\right) \rightarrow \Omega_{*}^{U}\left(A_{0}\right) \rightarrow \Omega_{*}^{U}(X) \rightarrow 0
$$

is exact.

With the lemma in hand, it is entirely straightforward to show that the arguments of $[7, \S 3]$ for finite complexes carry over to highly-connected $\mathrm{CW}$-spectra and thus apply to all $\mathrm{CW}$-complexes.

It remains to prove the lemma, and there is no loss of generality in supposing that $X$ is $(-1)$-connected. Then the proof can be easily extracted from Lemmas 18 and 19 and Example (iv) on p. 29 of [1, Lecture 1]. It is only necessary to insist in Lemma 18 that the finite subcomplex $X^{\prime}$ have dimension $\leqq p$, and to then choose $E_{\alpha}$ to be the $p$-skeleton of $E=M U$; then $S^{p} \wedge D E_{\alpha}$ has the homotopy type of a finite complex of dimension $\leqq p$, and so is $(-1)$-connected. Now Lemma 19 yields the desired cofibration.

Remark 3. For a finite group $G$, let $\operatorname{rank}(G)$ denote the largest integer $k$ such that $G$ contains an abelian subgroup with minimum number of generators $k$. I conjecture that

$$
\text { hom } \operatorname{dim}_{\Omega_{*} \Omega_{*}^{U}}^{U}\left(B_{G}\right)=\operatorname{rank}(G) .
$$

For example, we know that $\operatorname{rank}(G)=1$ if and only if hom 
$\operatorname{dim}_{\Omega_{*}} \Omega_{*}^{U}\left(B_{G}\right)=1$. (If $\Omega_{*}^{U}\left(B_{G}\right)$ is a projective module then $H_{*}\left(B_{G} ; Z\right.$ ) is free abelian, hence $\widetilde{H}_{*}\left(B_{G} ; \boldsymbol{Z}\right)=0$ and so $G$ is trivial.) If $G$ is elementary abelian the results of $[7, \S 5]$ show that

$$
\text { hom } \operatorname{dim}_{\Omega} v \Omega_{*}^{U}\left(B_{G}\right) \geqq \operatorname{rank}(G),
$$

but even in this case it is not generally known if $\Omega_{*}^{U}\left(B_{G}\right)$ has finite projective dimension.

Our final result concerns the relation between $\Omega_{*}^{U}\left(B_{G}\right)$ and $\Omega_{*}^{U}\left(B_{H}\right)$ when $H$ is a Sylow p-subgroup of $G$. In this situation there is the following diagram of induced homomorphisms and transfer homomorphisms $[5, \S 20]$ :

$$
\begin{array}{ccccc}
\Omega_{n}^{U}\left(B_{G}\right) & \stackrel{t}{\rightarrow} & \Omega_{n}^{U}\left(B_{H}\right) & \stackrel{i}{\rightarrow} & \Omega_{n}^{U}\left(B_{G}\right) \\
\downarrow \mu & & \downarrow \mu & & \downarrow \mu \\
H_{n}\left(B_{G} ; Z\right) & \stackrel{t}{\rightarrow} & H_{n}\left(B_{H} ; Z\right) & \stackrel{i}{\rightarrow} & H_{n}\left(B_{G} ; Z\right) .
\end{array}
$$

Let $\Omega_{n}^{U}\left(B_{G}\right)_{(p)}$ and $H_{n}\left(B_{G} ; \boldsymbol{Z}\right)_{(p)}$ denote the $p$-primary parts. It is well known that on $H_{n}\left(B_{G} ; \boldsymbol{Z}\right)$ the composition $i \circ t$ is multiplication by the index of $H$ in $G$. Since this index is prime to $p$, it follows that $\widetilde{H}_{n}\left(B_{H} ; \boldsymbol{Z}\right) \stackrel{i}{\rightarrow} H_{n}\left(B_{G} ; \boldsymbol{Z}\right)_{(p)}$ is a split epimorphism.

Proposition 6. Let $H$ be a Sylow p-subgroup of the finite group $G$. Then $i: \tilde{\Omega}_{*}^{U}\left(B_{H}\right) \rightarrow \Omega_{*}^{U}\left(B_{G}\right)_{(p)}$ is a split epimorphism of $\Omega_{*}^{U}$-modules.

Proof. It suffices to show that $i \circ t$ is an automorphism of $\Omega_{*}^{U}\left(B_{G}\right)_{(p)}$. Both $i$ and $t$ induce morphisms of spectral sequences, yielding the following composition on the $E^{2}$-terms:

$$
H_{*}\left(B_{G} ; \Omega_{*}^{U}\right) \stackrel{t}{\rightarrow} H_{*}\left(B_{H} ; \Omega_{*}^{U}\right) \stackrel{i}{\rightarrow} H_{*}\left(B_{G} ; \Omega_{*}^{U}\right) .
$$

Since this restricts to an isomorphism on the p-primary parts, it follows that $i \circ t$ yields an isomorphism on the $p$-primary parts of the $E^{\infty}$-terms. Since the complex bordism spectral sequence is convergent, it follows that $i \circ t$ is an automorphism of $\Omega_{*}^{U}\left(B_{G}\right)_{(p)}$.

REMARK 4. It follows that the projective dimension of the $\Omega_{*-}^{U}$ module $\Omega_{*}^{U}\left(B_{G}\right)$ is bounded by the maximum of the projective dimensions of the modules $\Omega_{*}^{U}\left(B_{H}\right)$ as $H$ runs through the Sylow subgroups of $G$. Of course the rank of $G$ is the maximum of the ranks of the Sylow subgroups of $G$. If the conjecture (3) holds for p-groups, and if

hom $\operatorname{dim}_{\Omega_{*}}^{U} \Omega_{*}^{U}\left(B_{H}\right) \leqq$ hom $\operatorname{dim}_{\Omega_{*}^{U}} \Omega_{*}^{U}\left(B_{G}\right)$ 
whenever $H$ is a subgroup of $G$, then the conjecture is true in general. I would very much like to know an upper bound to hom $\operatorname{dim}_{\Omega_{*}} \Omega_{*}^{U}\left(B_{G}\right)$ for $p$-groups.

\section{REFERENCES}

1. J. F. Adams, Lectures on generalized homology theories, Lecture Notes in Math., no. 99, Springer-Verlag, Berlin and New York, 1969.

2. M. F. Atiyah, Characters and cohomology of finite groups, Inst. Hautes Etudes Sci. Publ. Math. No. 9 (1961), 23-64. MR 26 \#6228.

3. J. M. Boardman, Stable homotopy theory, University of Warwick, 1965 (preprint).

4. H. Cartan and S. Eilenberg, Homological algebra, Princeton Univ. Press, Princeton, N. J., 1956. MR 17, 1040.

5. P. E. Conner and E. E. Floyd, Differentiable periodic maps, Ergebnisse der Mathematik und ihrer Grenzgebiete, Band 33, Academic Press, New York; SpringerVerlag, Berlin, 1964. MR 31 \#750.

6. - The relation of cobordism to K-theories, Lecture Notes in Math., no. 28, Springer-Verlag, Berlin and New York, 1966. MR 35 \#7344.

7. P. E. Conner and L. Smith, On the complex bordism of finite complexes, Inst. Hautes Etudes Sci. Publ. Math. No. 37 (1970).

8. T. tom Dieck, Bordism of G-manifolds and integrality theorems, Topology (to appear).

9. D. Lehmann, Sur le bordisme equivariant, C. R. Acad. Sci. Paris Ser. A-B 268 (1969), A693-A695.

10. R. G. Swan, Minimal resolutions for finite groups, Topology 4 (1965), 193-208. MR 31 \#3482.

11. - Groups with no odd dimensional cohomology, J. Algebra (to appear).

12. J. W. Vick, Pontrjagin duality in K-theory, Proc. Amer. Math. Soc. 24 (1970), 611-616.

Yale University, New Haven, Connecticut 06520 